\title{
ROTEIRO DE ATIVIDADES EM AMBIENTES VIRTUAIS DE APRENDIZAGEM PARA MEDIAÇÃO DE TRABALHOS DE CONCLUSÃO DE CURSO
}

Natana Lopes Pereira - PPGTIC/UFSC - natanapereiralopes@ hotmail.com Angelita Darela Mendes - PPGTIC/UFSC - angelita.mendes@ufsc.br Fernando José Spanhol - PPGTIC/UFSC - fernando.spanhol@ufsc.br

Resumo. A dinâmica social contemporânea impõe a adaptação do contexto educacional no que respeita a flexibilidade do acesso e a diversidade das formas de apresentação dos conteúdos. Este artigo tem como objetivo demonstrar o potencial mediador do Ambiente Virtual de Aprendizagem - AVA associado ao roteiro de atividades na estruturação de trabalho monográfico. A realização desta pesquisa, de abordagem qualitativa e caracterizada como um estudo de caso, compreendeu a elaboração de diversas atividades aplicadas no AVA - Moodle do curso Pós TIC SENASP. Para coleta de dados utilizou-se formulário eletrônico estruturado com questões de múltipla escolha. A partir da análise dos dados coletados conclui-se que a aplicação das atividades propostas mediadas pelo AVA contribuiu no processo de elaboração de TCC. Cabe uma discussão sobre aplicação de métodos de Gestão do Conhecimento - GC nesses ambientes e exploração de suas diversas ferramentas por meio do modelo SECI para melhor atender as necessidades dos acadêmicos.

Palavras-chave: Ambiente Virtual de Aprendizagem, Trabalho de Conclusão de Curso, Roteiro de Atividades

\section{SCRIPT OF ACTIVITIES IN VIRTUAL LEARNING ENVIRONMENTS FOR MEDIATION OF COURSE CONCLUSION WORKS}

Abstract. The contemporary social dynamic imposes the adaptation of the educational context regarding the flexibility of the access and the diversity of the forms of presentation of the contents. This article aims to demonstrate the potential mediator of the Virtual Learning Environment - VLE associated to the script of activities in the structuring of monographic work. The accomplishment of this research, of qualitative approach and characterized as a case study, comprised the elaboration of several activities applied in the VLE - Moodle - of the course Pos TIC SENASP. For data collection, a structured electronic form with multiple choice questions was used. From the analysis of the collected data, it is concluded that the application of the proposed activities mediated by VLE contributed in the process of elaboration of Course Conclusion Works. There is a discussion about the application of Knowledge Management (KM) methods in these environments and the exploration of their various tools through the SECI model to better meet the needs of academics.

Keywords: Virtual Learning Environment, Course Conclusion Works, Script Of Activities

\section{Introdução}

Dados do Indicador de Alfabetismo no mundo do trabalho apontaram as habilidades com leitura, escrita e matemática de pessoas entre 15 e 64 anos pelo Instituto Paulo Montenegro, em 2016. Essa pesquisa identificou que apenas 8\% das pessoas com ensino superior possuem o nível considerado proficiente, o mais alto na escala, como elaboração de textos mais complexos, interpretação de tabelas, gráficos e 
resolução de problemas. Em 2014, o índice de letramento científico - ILC pesquisa realizada em nove regiões metropolitanas, com pessoas entre 15 e 40 anos e com mais de quatro anos de estudo, indicou que $48 \%$ dessas pessoas foram classificadas no nível de letramento científico rudimentar e apenas 5\% classificadas no nível de letramento cientifico proficiente. Essas pesquisas são realizadas no âmbito dos estudos do letramento (STREET, 1984, KLEIMAN, 1995), cujo objeto primordial é entender e descrever como se dá a compreensão e o uso da leitura pela sociedade.

A corroborar esses dados, verifica-se que a elaboração e entrega de trabalho monográfico apresenta-se como um dos maiores motivos de desistências tanto no ensino a distância como no ensino presencial. Muitas vezes a dificuldade de contato presencial com o orientador, a falta de planejamento e consequentemente uma estrutura mal organizada são alguns dos principais motivos de desistências dos cursos de graduação e pós-graduação (SILVA; SIHRLEI; SILVA, 2012). Quando se incluem variáveis como o retorno aos estudos depois de anos afastado e tempo para o estudo compartilhado com o tempo de trabalho, as dificuldades aumentam e estratégias precisam ser estabelecidas por alunos e professores para a conclusão da etapa final, o Trabalho de Conclusão de Curso - TCC e obtenção de sucesso acadêmico.

O Ambiente Virtual de Aprendizagem - $\mathrm{AVA}^{1}$ é um recurso tecnológico amplamente utilizado na educação a distância. Ele possibilita a mediação do processo de aprendizagem, flexibiliza o acesso e diversifica enormemente os materiais, permitindo atendimento às características e lacunas de aprendizagem de cada aluno. $\mathrm{O}$ aprimoramento dos recursos de Tecnologias da Informação e Comunicação - TIC disponibilizados nesse ambiente e o aperfeiçoamento de técnicas de ensino e de aprendizagem (roteiro, atividades, suporte virtual e/ou presencial) podem incrementar e otimizar a execução de processos em que a comunicação presencial seria um grande facilitador, como a elaboração de TCC (MACHADO et al., 2014a).

Nesse contexto, o trabalho proposto objetiva apresentar um roteiro de atividades mediado pelo AVA que possibilite maior interação entre orientando e orientador por meio de suas ferramentas de comunicação e o planejamento e estruturação de TCC. Para atender tal objetivo, realizou-se um estudo de caso na pós-graduação (lato sensu) em Tecnologias da Informação e Comunicação aplicadas à Segurança Pública e Direitos Humanos - Pós TIC SENASP da Universidade Federal de Santa Catarina - UFSC que ocorre em parceria com o Ministério da Justiça - MJ. O curso, de modalidade presencial, tem como público alvo profissionais de segurança pública da ativa. Visando atender às necessidades dos acadêmicos utilizou-se como ferramenta de suporte ao ensino presencial o Ambiente Virtual de Aprendizagem - Moodle. De acordo com Silva (2011) essa plataforma é utilizada pela UFSC, tanto no ensino a distância como presencial, sendo um dos motivos de sua utilização além de ser uma plataforma livre possuir diversos recursos de TIC que contribuem no processo de ensino-aprendizagem.

\section{Moodle - Um Ambiente Virtual para o Ensino e Aprendizagem}

Em 1999, Pierre Levy preconizava a necessidade de flexibilização do formato e da oferta de educação formal, além do atendimento às especificidades em termos de trajetórias pessoais, portanto, exigência de diferentes conhecimentos para pessoas diferentes, impondo a necessidade de se repensar a organização do currículo, das metodologias, bem como dos tempos e dos espaços de aprendizagem. $\mathrm{O}$ avanço tecnológico, no que respeita o domínio e o acesso ao conhecimento, impôs uma realidade para a educação que demanda crescente diversificação e personalização da oferta educacional. O aprimoramento das ferramentas tecnológicas tornou mais flexível 
as atividades educacionais, podendo ser adaptadas de acordo com o perfil do aluno (LACERDA, 2013).

Para Lacerda (2013), o desenvolvimento de novas ferramentas de interatividade da Web $2.0^{2}$ e sua utilização no AVA ampliou sobremaneira a utilização desses ambientes, que possuem como principal característica o gerenciamento e a possibilidade de construção individual ou colaborativa do conhecimento por mediação das TIC. Por meio de várias ferramentas disponíveis e de uma rede de computadores interligados foi possível a "virtualização" do processo de ensino-aprendizagem.

Para Pereira (2007apud MCKIMM; JOLLIE; CANTILLON, 2003) o AVA é um conjunto de ferramentas eletrônicas que compõem um Sistema de Gestão de Aprendizagem - $S G A^{3}$, organizadas em quatro eixos: Informação e Documentação; Comunicação; Gerenciamento Pedagógico; Produção. Esses itens permitem organizar conteúdos, acompanhar atividades, suporte on-line e comunicação eletrônica por meio de diversos recursos de TIC disponibilizadas pela plataforma.

A plataforma Moodle - Modular Objetc Oriented Dynamic Learning Environmet é um AVA que vem sendo cada vez mais aderido pelas instituições de ensino para gerenciamento de cursos. Silva (2011) o caracteriza por ser um ambiente on-line de aprendizagem Open Source, criado por Martin Dougiamas, e trabalha com uma perspectiva dinâmica de aprendizagem dando ênfase às ações colaborativas. $\mathrm{Na}$ mesma concepção do autor o objetivo desse ambiente é proporcionar ensinoaprendizagem por meio de interação entre os usuários.

De acordo com Saldanha (2011) o Moodle objetiva cobrir três (3) eixos básicos do processo de ensino-aprendizagem sendo eles: Gerenciamento de conteúdos por meio da organização dos temas disponibilizados aos alunos de acordo com a disciplina; Interação entre usuários com a utilização de diversas ferramentas que possibilitam a comunicação síncrona (tempo real) e assíncrona (tempo distinto) entre os atores envolvidos; Acompanhamento e Avaliação possibilitadas por relatórios, avaliação de tarefas, atribuição de notas, entre outros.

Esses itens, supracitados, são importantes para a configuração de um curso, permitindo por meio de diversos recursos a disponibilização de materiais de estudo no AVA, proporcionando ao usuário um ambiente colaborativo, interativo e propício ao processo de ensino-aprendizagem (SILVA, 2011). Lacerda (2013) agrupa as diversas ferramentas que auxiliam no gerenciamento de aprendizagem e construção do conhecimento conforme figura seguir:

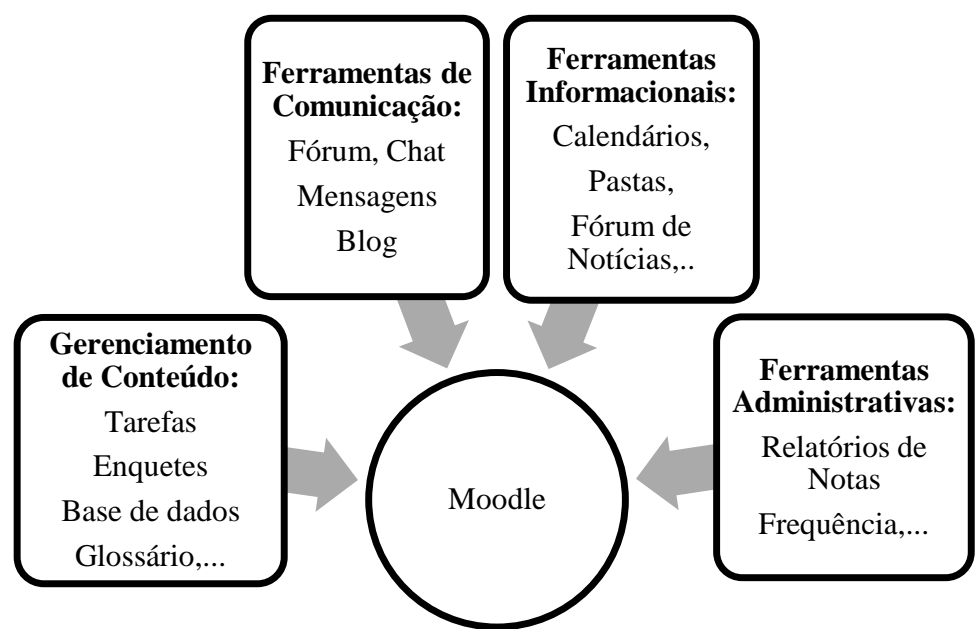

Figura1:Ferramentas Moodle - Adaptado Lacerda A. L (2013). 
O objetivo principal do Moodle elencado por Vicentin (2007) é o gerenciamento e a disseminação da aprendizagem. Em um contexto de cultura digital pode ser utilizado como uma ferramenta para construção de comunidades colaborativas, proporcionando a construção do conhecimento por meio da colaboração, interação e participação de um grupo de pessoas utilizando principalmente os diversos recursos de comunicação síncrona e assíncrona (VICENTIN, 2007).

\section{Métodos Utilizados na Pesquisa}

Esta pesquisa apresenta um estudo de caso realizado no curso Pós TIC SENASP sobre a aplicação de um roteiro de atividades no AVA da disciplina de Metodologia da Pesquisa Científica para estruturação de TCC. Freire (2013) caracteriza o estudo de caso, como um estudo aprofundado e exaustivo para identificar a aplicabilidade de uma teoria, suas limitações e diferenças. Para melhor compreensão do estudo realizado utilizou-se a abordagem qualitativa que, de acordo Freire (2013), visa analisar a interação das variáveis envolvidas no estudo, compreender e classificar o processo envolvendo grupos sociais e contribuir no processo de mudança de determinado grupo.

Para a avaliação da experiência com o roteiro de atividades, utilizou-se como instrumento de coleta de dados um formulário eletrônico composto por questões de múltipla escolha, adotando a escala Likert composta por cinco (5) níveis. De acordo com Rocha (2011), a escala Likert foi proposta por Rensis Likert, em 1932, combinando um conjunto de respostas em relação ao objeto pesquisado, em que os respondentes são solicitados a informar o grau de concordância ou discordância das afirmações.

Nos procedimentos para realização do estudo utilizou-se a pesquisa bibliográfica. A pesquisa bibliográfica, realizada por meio de leitura e análise de periódicos e livros, segundo Gil (2010) é desenvolvida mediante material já elaborado, principalmente livros, artigos científicos, revistas, teses, entre outros.

O público alvo deste estudo compreende profissionais de segurança pública da ativa, dispondo de pouco tempo para orientação presencial devido horários alternados, plantões, entre outros. Também se caracteriza pela distinta faixa etária, contendo discentes que concluíram o ensino superior recentemente, como por exemplo, em 2013 e 2014, até discentes que estão a mais de dezessete (17) anos afastados do processo de escrita científica, sendo um agravante para a elaboração de trabalhos acadêmicos segundo Antunes et al. (2011).

\subsection{Estrutura e Aplicação do Roteiro Proposto}

O modelo de roteiro desenvolvido utilizou como principal ferramenta o Ambiente Virtual de Aprendizagem - Moodle. A partir de embasamento teórico dos autores Marconi e Lakatos (2010), Freire (2013) e Gil (2010), elaborou-se um modelo composto por oito (8) etapas para estruturação de monografias mediadas pelas ferramentas de TIC da plataforma. O processo de interação a cada execução das etapas ocorreu de três (3) modos, tendo como base a classificação de Machado et al. (2014b apud LÉVY,1999): Muitos para muitos: Atividades em fóruns abertas sem restrições de grupos, possibilitando interação entre os mesmos; Um para muitos: Fóruns com acesso restrito ao orientador e seus respectivos orientandos; Um para um: Fóruns com acesso restrito do orientador e o orientando, individualmente. 
Quadro 1: Etapas Roteiro de Atividades

\begin{tabular}{|c|c|c|c|}
\hline Etapa & Descrição & Atividades & Recursos/Ferramentas \\
\hline $1^{\text {a }}$ Etapa & $\begin{array}{c}\text { Identificação: Corpus do Objeto } \\
\text { e Problema da Pesquisa }\end{array}$ & Tarefa & $\begin{array}{l}\text { Rótulos, páginas de texto simples, } \\
\text { links, recursos de vídeo. }\end{array}$ \\
\hline $2^{\text {a }}$ Etapa & Definição de Orientação & $\begin{array}{l}\begin{array}{c}\text { Fórum em } \\
\text { grupo }\end{array} \\
\end{array}$ & Pastas, link para arquivos. \\
\hline $3^{\text {a }}$ Etapa & $\begin{array}{l}\text { Aperfeiçoamento: Introdução, } \\
\text { Justificativa, Objetivos do } \\
\text { Estudo. Indicação Bibliográfica }\end{array}$ & $\begin{array}{l}\text { Fórum em } \\
\text { grupos }\end{array}$ & $\begin{array}{c}\text { Rótulos, páginas de texto simples, } \\
\text { link para arquivos, recurso de } \\
\text { agrupamento. }\end{array}$ \\
\hline $4^{\text {a }}$ Etapa & $\begin{array}{c}\text { Delineamento e Organização do } \\
\text { Instrumental de Pesquisa }\end{array}$ & $\begin{array}{l}\text { Fórum em } \\
\text { grupos }\end{array}$ & Rótulos, link para arquivos. \\
\hline $5^{\mathbf{a}}$ Etapa & $\begin{array}{l}\text { Alinhamento e Revisão } \\
\text { Metodológica }\end{array}$ & $\begin{array}{l}\text { Fórum } \\
\text { individual }\end{array}$ & $\begin{array}{l}\text { Rótulos, páginas de texto simples, } \\
\text { link para arquivos. }\end{array}$ \\
\hline 6 ${ }^{\text {a }}$ Etapa & Coleta de Dados & $\begin{array}{l}\text { Fórum } \\
\text { individual }\end{array}$ & $\begin{array}{l}\text { Rótulos, páginas de texto simples, } \\
\text { link para arquivos. }\end{array}$ \\
\hline $7^{\text {a }}$ Etapa & $\begin{array}{c}\text { Análise e Representação dos } \\
\text { Dados Coletados }\end{array}$ & $\begin{array}{l}\text { Fórum } \\
\text { individual }\end{array}$ & $\begin{array}{l}\text { Rótulos, páginas de texto simples, } \\
\text { link para arquivos. }\end{array}$ \\
\hline $8^{\text {a }}$ Etapa & $\begin{array}{c}\text { Conclusão da Estruturação dos } \\
\text { itens da Monografia }\end{array}$ & Tarefa & Rótulos, páginas de texto simples. \\
\hline
\end{tabular}

Fonte: Desenvolvido pelo (s) autor (es).

A primeira etapa do roteiro apresentado refere-se aos seguintes itens de pesquisa: identificação do corpus da pesquisa, do objeto da pesquisa e problema da pesquisa. A atividade proposta objetivou elaboração de itens de pesquisa elencados por Freire (2013): Tema, Problema, Pergunta de Pesquisa, Objetivos, Hipóteses e Justificativas. Essa atividade teve como objetivo principal instigar o aluno à pesquisa e influenciá-lo a refletir sobre seu cotidiano, desenvolvendo um olhar de pesquisador, analisando problemas encontrados em seu dia a dia e buscando, apontando possíveis soluções por meio das TIC. O recurso do Moodle utilizado para execução dessa etapa foi a "Tarefa". Essa ferramenta, segundo Silva (2011), possibilita diversas atividades como a produção off-line, on-line e envio de arquivos.

Na etapa Definição de Orientação (segunda etapa), após coleta de todos os préprojetos de pesquisa, os mesmos foram disponibilizados aos professores por meio da ferramenta do Moodle "Pastas". Segundo Silva (2011) ela possibilita a inserção de arquivos em pastas distintas facilitando a organização dos mesmos. Para facilitar a comunicação na definição de orientação, acrescentou-se no Ambiente Virtual de Aprendizagem um fórum de acesso restrito ao grupo "professores".

Após definição das orientações, iniciou-se a terceira etapa referente à implementação e ao aperfeiçoamento da introdução, justificativa, problema de pesquisa, objetivos: geral e específicos, e também a indicação de referência bibliográfica. Para esse processo de comunicação optou-se pela ferramenta fórum. Essa ferramenta apresenta como funções em um AVA: o intercâmbio de informações, tais como o diálogo, o debate e a comunicação; espaço de socialização; trabalho e aprendizagem colaborativos (SALDANHA; 2011 apud SANCHÉZ; 2005). Nesse recurso, foram criados grupos compostos pelo orientador e seus respectivos orientandos. 
Cada fórum para comunicação com o orientador foi restrito aos grupos criados, impossibilitando acesso aos não participantes dos mesmos.

A definição da metodologia para execução da pesquisa ocorreu na quarta etapa da disciplina. Esse item é extremamente importante no processo de elaboração e construção de um TCC. A definição imprecisa dos métodos abordados no trabalho a ser elaborado pode acarretar diversos problemas ao pesquisador que, segundo Freire (2013), deve estudar sobre os diversos procedimentos para levantamento, tabulação e análise de dados e escolher quais os levarão a alcançar o objetivo da pesquisa. Nessa etapa, além de disponibilizar sugestões bibliográficas (GIL; MARCONI; LAKATOS, 2010) aplicou-se atividade referente à elaboração dos procedimentos metodológicos que os alunos utilizariam em suas pesquisas. Essa tarefa teve como objetivo a construção de conhecimento sobre metodologia científica.

$\mathrm{Na}$ quinta etapa, pode-se alinhar e realizar revisão metodológica, segundo Marconi e Lakatos (2010), a qual define a expressão "como" de sua pesquisa. Essa fase envolveu os processos de localizar, analisar, sintetizar e interpretar diversos conteúdos relacionados à área de estudo abrangida. $\mathrm{O}$ tópico de estudo proposto ocorreu de forma presencial, abrangendo os tipos de revisão da literatura. Além dos tipos de revisão de literatura, foram apresentadas diversas plataformas de base de dados, visando auxiliar o processo de pesquisa do aluno. A partir dessa etapa, para maior aproximação dos atores envolvidos, foram desenvolvidos fóruns de comunicação individual de modo um para um (orientando/orientador), em que o aluno deveria postar seu trabalho estruturado até o item: fundamentação teórica.

A sexta etapa incentivou o aluno, de acordo com o conhecimento já adquirido nas etapas anteriores, a planejar o instrumento e o modelo de coleta de dados que iria aplicar, realizar testes, entre outros. A execução de coleta de dados não se refere somente a aplicação de questionários, segundo Marconi e Lakatos (2010) há vários procedimentos para a realização de coleta de dados como pesquisa bibliográfica, entrevistas, testes, formulários, entre outros. Após o planejamento da coleta de dados, foram inseridas algumas orientações para tratamento dos dados coletados. Nessas etapas, foram utilizados os recursos de "rótulo", "páginas de texto simples" e a disponibilização de arquivos, visando auxiliar o aluno na implementação dos itens abordados no período correspondente à elaboração e desenvolvimento da monografia (janeiro à maio de 2017).

A última atividade (conclusão da estruturação dos itens da monografia) do roteiro proposto utilizou o recurso "Tarefa" do Moodle. A atividade consistia na implementação dos itens que irão constituir o desenvolvimento da monografia e implementar os seguintes itens que compõem o resumo: Assunto/ Focalizaçãa Temática, Intencionalidade (Objetivo da pesquisa e pergunta da pesquisa), Metodologia, Resultado, Discussão e Palavras-chave. Segundo Marconi e Lakatos (2010) o resumo contém todas as informações principais do texto abrangendo os objetivos e o assunto, os métodos e as técnicas, os resultados e as conclusões. Ele contém a definição clara e objetiva dos itens mais importantes da pesquisa.

\section{Apresentação e Discussão dos Resultados}

A pesquisa buscou analisar, por meio da aplicação de um roteiro de atividades o AVA como elemento mediador na estruturação de TCC. O instrumento de coleta de dados foi enviado aos doze (12) professores/orientadores e aos 27 alunos do curso. Obteve-se participação de dez (10) docentes e dezenove (19) discentes. 
A pesquisa, inicialmente, buscou analisar a participação e aderência às atividades propostas. De acordo com os dados coletados, $89 \%$ dos discentes informaram participar das atividades elaboradas na disciplina de Metodologia da Pesquisa Científica e apenas $11 \%$ não opinaram.

A ferramenta mais utilizada no AVA para aplicação do roteiro de atividades foi o recurso "fórum". Na avaliação, pode-se analisar que $69 \%$ dos alunos utilizaram essa ferramenta para iniciar o contato com o orientador (21\% concordaram totalmente e $48 \%$ concordaram). Não opinaram $26 \%$ e $5 \%$ dos alunos discordaram. Confirmando esses dados a análise de relatórios de participação (gerenciamento) do Moodle, indicou participação de aproximadamente $72 \%$ dos discentes na ferramenta fórum, e registrando em média aproximadamente o envio de sete (7) à oito (8) mensagens por orientando. Segundo Silva (2011), o fórum é caracterizado pela possibilidade de envio de anexos, pela interação de forma assíncrona e pela troca de conhecimento. Apresenta como funções o intercâmbio de informações, tais como o diálogo, o debate e a comunicação; espaço de socialização; trabalho e aprendizagem colaborativos (SALDANHA; 2011 apud SANCHÉZ; 2005).

Tendo como público alvo do curso profissionais de segurança pública, questionou-se sobre a interferência da atuação profissional no desempenho das etapas do roteiro aplicado. A pesquisa demonstrou que $95 \%$ dos alunos informaram que a mesma interferiu de forma negativa em seu desempenho nas atividades da disciplina e $5 \%$ informaram que não houve interferência da atuação profissional. Mesmo apresentando esse agravante o AVA foi utilizado por $89 \%$ dos discentes na mediação para estruturação da monografia.

Buscando poder mensurar a interferência do roteiro de atividades mediado pelo AVA, questionou-se sobre a possível contribuição do ambiente nesse processo. Aproximadamente $80 \%$ dos professores e 63,2\% dos acadêmicos confirmaram a contribuição do AVA e do roteiro de atividades no processo de estruturação de monografias. A abstenção de aproximadamente $36 \%$ dos discentes e $20 \%$ docentes é explicada devido a uma dificuldade encontrada nesse processo, o fator tempo. A interferência da variável "tempo" também foi identificada por Antunes et al. (2011) como uma das dificuldades para elaboração de TCC.

Gráfico1:Avaliação Roteiro de Atividades

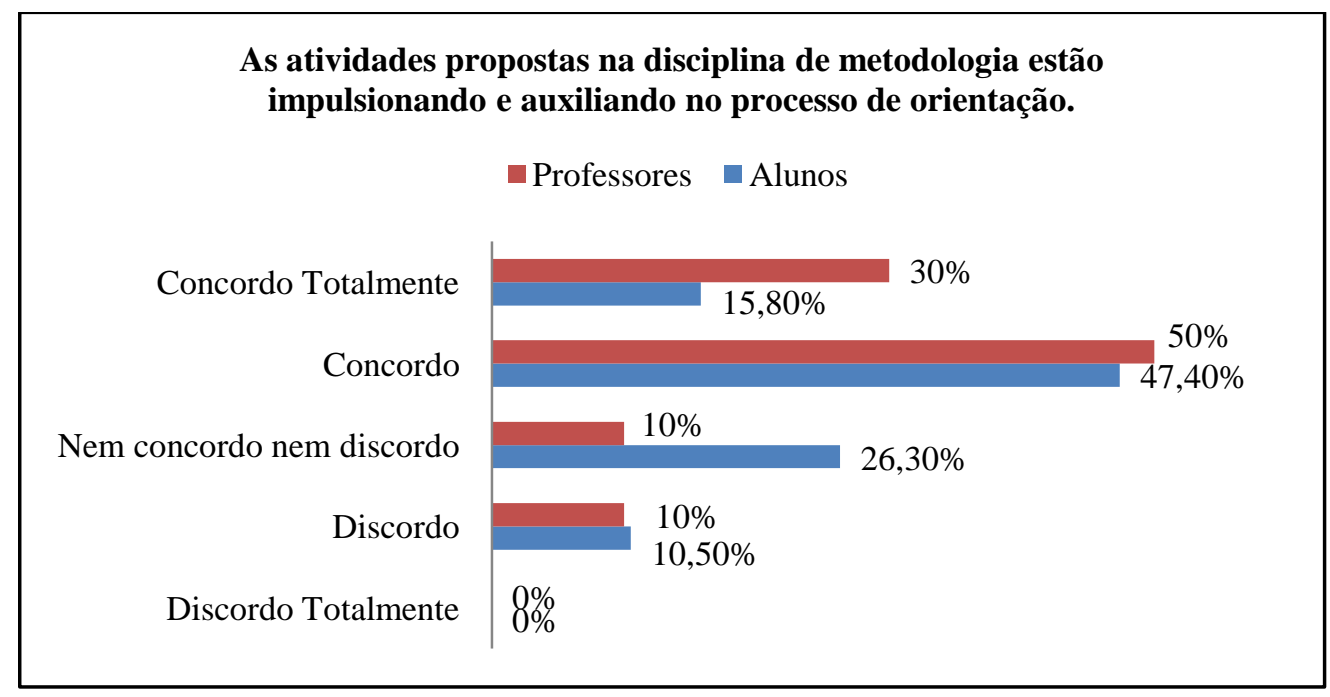

Fonte: Desenvolvido pelo (s) autor(es).

A partir da análise dos dados coletados dos professores e dos alunos pode-se constatar que o roteiro de atividades mediado pelo AVA auxilia na estruturação de TCC 
e na interação entre os atores envolvidos. Por meio das atividades realizadas no Moodle os alunos planejaram, especificaram os principais itens de suas pesquisas e identificaram métodos a utilizar para elaboração da mesma.

\section{Considerações Finais}

Segundo Machado et al. (2014b), a comunicação é a principal "ferramenta" para a orientação de TCC em Ead, sendo necessário utilizar as TIC para auxiliar nesse processo. Após a execução do estudo de caso e realização de análise dos dados coletados, pode-se confirmar a hipótese criada, neste trabalho, sobre a contribuição do AVA no processo de estruturação de TCC no projeto de especialização para o público previamente definido, como forma de capacitação desse grupo.

Bastos et al.(2016) corroboram a importância do planejamento e utilização de recursos tecnológicos no processo de mediação de TCC. É de extrema importância a utilização das TIC para impulsionar o aluno a planejar os métodos de pesquisa, a interagir frequentemente com seu orientador, construindo por etapas seu TCC e tornando o processo menos cansativo uma vez que a delimitação dos "passos" facilita nessa "longa caminhada", no desenvolvimento de seu trabalho final.

Em estudos realizados por Antunes et al. (2011) evidencia-se a preocupação em otimização do processo de orientação, devido a escassez de tempo, por meio da elaboração do projeto de pesquisa na disciplina de metodologia da pesquisa e o estabelecimento de ferramentas de apoio e monitoramento. Confirmando esses dados a pesquisa demonstrou o impacto do fator tempo e a dificuldade da escrita uma vez que alguns alunos estão a mais de dezessete (17) anos afastados do processo de produção textual acadêmico. Assim enfatiza-se a importância do roteiro elaborado mediado pelo AVA, que por meio da disciplina de metodologia de pesquisa científica, proporcionou ensino-aprendizagem em métodos para pesquisa, planejou antecipadamente as etapas para elaboração dos itens de pesquisa (estruturação) da monografia e incentivou a comunicação para orientação, sendo esses primordiais para a elaboração e desenvolvimento de TCC.

Com base na análise dos dados e estendendo a discussão para trabalhos futuros, além da contribuição do AVA nesse processo, identificou-se a necessidade de aperfeiçoar por meio desses ambientes o processo (método) de interação entre os protagonistas envolvidos (orientando e orientador) e a estruturação de TCC, utilizando métodos de Gestão do Conhecimento - GC. Segundo Dalkir (2005), a GC incorpora tanto a captura e armazenamento da perspectiva do conhecimento como a valorização dos ativos intelectuais. Inicialmente aplicada somente nas organizações, a GC busca por uma melhora em métodos e processos, proporcionando um ambiente que intensifique a construção e disseminação do conhecimento em trabalhos científicos por meio de pessoas, processo e tecnologia.

Cada vez mais, as práticas de GC vêm sendo aplicadas no contexto educacional por meio principalmente dos AVA. Neles são elaborados espaços colaborativos onde a construção e compartilhamento do conhecimento tanto tácito (conhecimento pessoal) como explícito (conhecimento registrado de forma tangível) é seu principal objetivo. Cabe estudo sobre a forma de criação e compartilhamento desse conhecimento por meio do AVA tendo como base os modos de conversão de conhecimento elaborado por Takeuchi e Nonaka (2009) modelo SECI - Socialização, Externalização, Combinação e Internalização, e por meio do estudo desses métodos nas ferramentas de TIC do AVA poder elencar boas práticas no processo de estruturação de trabalhos de conclusão de cursos mediadas por esses ambientes. 


\section{Agradecimentos}

À Fundação de Amparo à Pesquisa e Inovação do Estado de Santa Catarina - FAPESC

\section{Referências}

ANTUNES, Elaine di Diego et al. Desafios na Construção do Trabalho de Conclusão do Curso de Especialização em Negociação Coletiva / Modalidade a Distância. Renote Novas Tecnologias na Educação, v. 2, n. 9, maio 2011. Disponível em: $<$ http://www.seer.ufrgs.br/index.php/renote/article/view/25140/14625>. Acesso em: 03 jul. 2017.

BASTOS, Isis Maria Monteles et al. O uso de ferramentas de interação e comunicação na orientação de trabalhos de conclusão de cursos a distância. Revista Espacios, v. 22, n. 37, maio 2016. Disponível em: $<$ http://www.revistaespacios.com/a16v37n22/163722e2.html $>$.Acesso em: 01 jul. 2017.

DALKIR, Kimiz. Knowledge Management in Theory and Practice. Elsevier, 2005.

FREIRE, Patrícia de Sá. Aumente qualidade e quantidade de suas publicações científicas: Manual para elaboração de projetos e artigos científicos. 1. ed. - Curitiba, PR: CRV, 2013.

GIL, A. C. Como Elaborar Projetos de Pesquisa. São Paulo: Atlas, 2010.

KLEIMAN, A. Os significados do letramento: uma nova perspectiva sobre a prática social da escrita. Campinas: Mercado das letras, 1995.

LACERDA, A. L. CONTRIBUIÇÕES DO DESIGN INSTRUCIONAL AO ENSINO PRESENCIAL DE FÍSICA APOIADO POR AMBIENTE VIRTUAL DE APRENDIZAGEM. 2013. 246 f. Dissertação (Mestrado) - Curso de Educação Científica e Tecnológica, Universidade Federal de Santa Catarina, Florianópolis, 2013.

LEVY, P. Cibercultura. São Paulo: Editora 34, 1999.

MACHADO, Andrea de Bem et al. COMUNICAÇÃO NA EAD: UMA ANÁLISE NO PROCESSO DE ORIENTAÇÃO DE MONOGRAFIA. In: CIAED- CONGRESSO NACIONAL DE EDUCAÇÃO A DISTÂNCIA, 20, 2014a, Florianópolis. Anais. Disponível em: <http://www.abed.org.br/hotsite/20-ciaed/pt/anais/pdf/98.pdf>. Acesso em: 15 out. 2016.

MACHADO, Andreia de Bem et al. ORIENTAÇÃO NA EDUCAÇÃO A DISTÂNCIA: UMA ANÁLISE DA REALIDADE BRASILEIRA. Revista da Universidade Vale do Rio Verde, Três Corações, v. 2, n. 12, p.149-158, dez. 2014b.

MARCONI, A.M; LAKATOS, E. M. Fundamentos de metodologias científica. 2.ed. São Paulo: Atlas 2010.

PEREIRA, Alice T. Cybis. Ambientes Virtuais de Aprendizagem: Em diferentes contextos. Rio de Janeiro: Ciência Moderna Ltda, 2007. 210 p. 
ROCHA, Henrique Martins. Abordagem metodológica na análise de dados de estudos não- paramétricos, com base em respostas em escalas ordinais. Gepros, São Paulo, v. 3, n. 6, p.77-91, abr. 2011. Disponível em: <http://revista.feb.unesp.br/index.php/gepros/article/view/649/358>. Acesso em: 11 nov. 2016.

SALDANHA, Camila Teixeira. Interação nos Fóruns de Discussão: Uma análise Linguística. 2011. 201 f. Dissertação (Mestrado) - Curso de Pós-graduação em Educação, Universidade Federal de Santa Catarina, Florianópolis, 2011.

SILVA, R. S. Moodle para autores e tutores. 2. ed. São Paulo: Novatec Editora, 2011.

VICENTIN, Robson Luis. Um ambiente Virtual de Aprendizagem como Instrumento de Apoio ao Processo de Orientação em uma Pós- Graduação em Arquitetura: Estudo de caso PósARQ. 2007. 121 f. Dissertação (Mestrado) - Curso de Arquitetura e Urbanismo, Centro Tecnológico, Universidade Federal de Santa Catarina, Florianópolis, 2007.

SILVA, Ana Paula Costa e; SIHLER, Anelise Pereira; SILVA, Chris Alves da. ORIENTAÇÃO DE TRABALHOS DE CONCLUSÃO DE CURSO A DISTÂNCIA: UMA EXPERIÊNCIA FUNDAMENTADA NA INTERAÇÃO. Renote - Novas Tecnologias na Educação, UFRGS, v. 10, n. 1, p.1-07, jul. 2012. Disponível em: < http://www.seer.ufrgs.br/renote/article/download/30855/19211>. Acessoem: 15 maio 2016.

STREET, B. Literacy in theory and practice. Cambrigde, Cambrigde University Press, 1984.

TAKEUCHI, Hirotaka; NONAKA, Ikujiro. Gestão do Conhecimento. Porto Alegre: Bookman, 2009. 320 p.

URIARTE, Luiz Ricardo. Modelo de Ambiente para Orientação a Distância. 2003. 185 f. Tese (Doutorado) - Curso de Engenharia de Produção, Centro Tecnológico, Universidade Federal de Santa Catarina, Florianópolis, 2003.

\footnotetext{
${ }^{1}$ A sigla ou o termo AVA é utilizado neste trabalho como sinônimo de Ambiente Virtual de Ensino Aprendizagem- AVEA, abrangendo em seu entendimento o ensino-aprendizagem. De acordo com a concepção de Pereira (2007) o termo AVA é mais difundido no Brasil e objetiva proporcionar meios para que ocorra aprendizado ressaltando a importância de ensino nesse processo.

${ }^{2}$ WEB 2.0 - É uma segunda geração de comunidades e serviços, tendo como conceito a "Web como plataforma", envolvendo wikis, aplicativos baseados em, redes sociais, blogs e Tecnologia da Informação. Fonte: https://pt.wikipedia.org/wiki/Web_2.0.

${ }^{3}$ SGA -disponibiliza uma série de recursos, síncronos e assíncronos, que dão suporte ao processo de aprendizagem, permitindo seu planejamento, implementação e avaliação. Fonte: https://pt.wikipedia.org/wiki/Sistema_de_Gest\%C3\%A3o_da_Aprendizagem.
} 